\title{
UNA NUEVA TILLANDSIA (BROMELIACEAE) DE LA SIERRA DEL HALO, TECALITLÁN, JALISCO, MÉXICO
}

\author{
Adolfo Espejo Serna y Ana Rosa López-Ferrari \\ Universidad Autónoma Metropolitana-Iztapalapa, División de Ciencias Biológicas \\ y de la Salud, Departamento de Biología, Herbario Metropolitano, \\ 09340 México, D.F., México. aes@xanum.uam.mx
}

\section{RESUMEN}

Se describe e ilustra Tillandsia sierrahalensis, conocida del estado de Jalisco, México. Se compara la nueva especie con T. hintoniana L. B. Sm. que presenta características similares y se incluye un cuadro comparativo y un mapa de distribución de las dos especies.

Palabras clave: Bromeliaceae, Jalisco, México, Tillandsia.

\begin{abstract}
Tillandsia sierrahalensis known from the state of Jalisco, Mexico, is described and illustrated. The new species is compared with T. hintoniana L. B. Sm., species with similar characteristics and a compartive table and a distribution map of both taxa are included.
\end{abstract}

Key words: Bromeliaceae, Jalisco, Mexico, Tillandsia.

Como resultado de las exploraciones en el campo y de la revisión de material herborizado en diversas colecciones institucionales, con el fin de completar la Flora Bromeliológica Mexicana, se detectó material de una especie no descrita del género Tillandsia, que aquí proponemos como nueva.

Tillandsia sierrahalensis Espejo et López-Ferrari, sp. nov. Figs. 1 y 2

Herbae epiphyticae, acaules, rosulatae, florens usque ad $60 \mathrm{~cm}$ altae. Folia basalia, vaginae distinctae, oblongo-elipticae, $9.5-11 \mathrm{~cm}$ longae, $5.5-6 \mathrm{~cm}$ latae; laminae anguste triangulari-lanceolatae, $18-25 \mathrm{~cm}$ longae, $2-2.5 \mathrm{~cm}$ latae, erectae; 


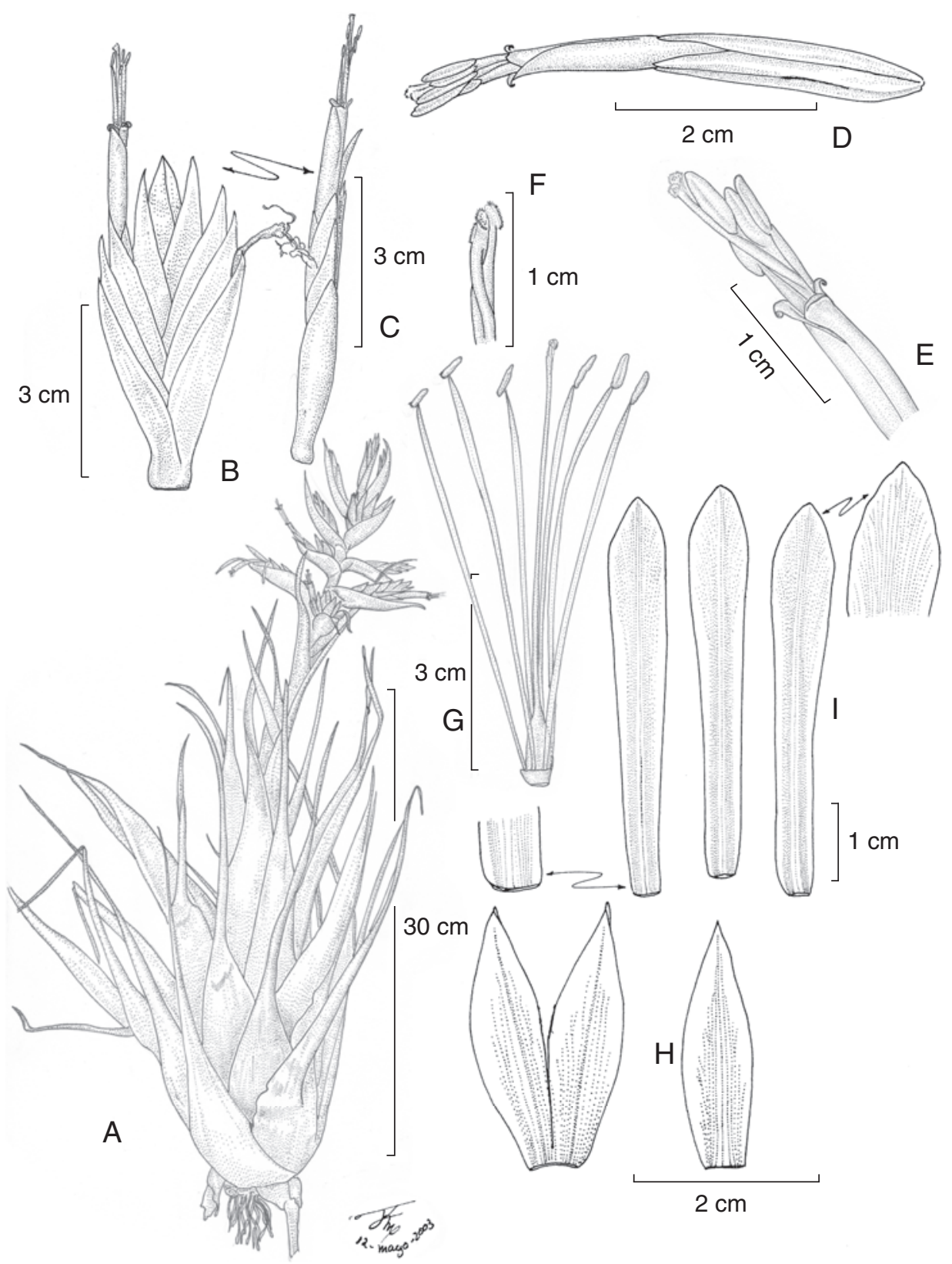

Fig. 1. Tillandsia sierrahalensis Espejo et López-Ferrari. A. hábito; B. espiga, vista frontal; C. espiga, vista lateral; D. flor; E. detalle de la porción apical de la flor; F. estigma; G. gineceo y androceo; H. sépalos; I. pétalos. 

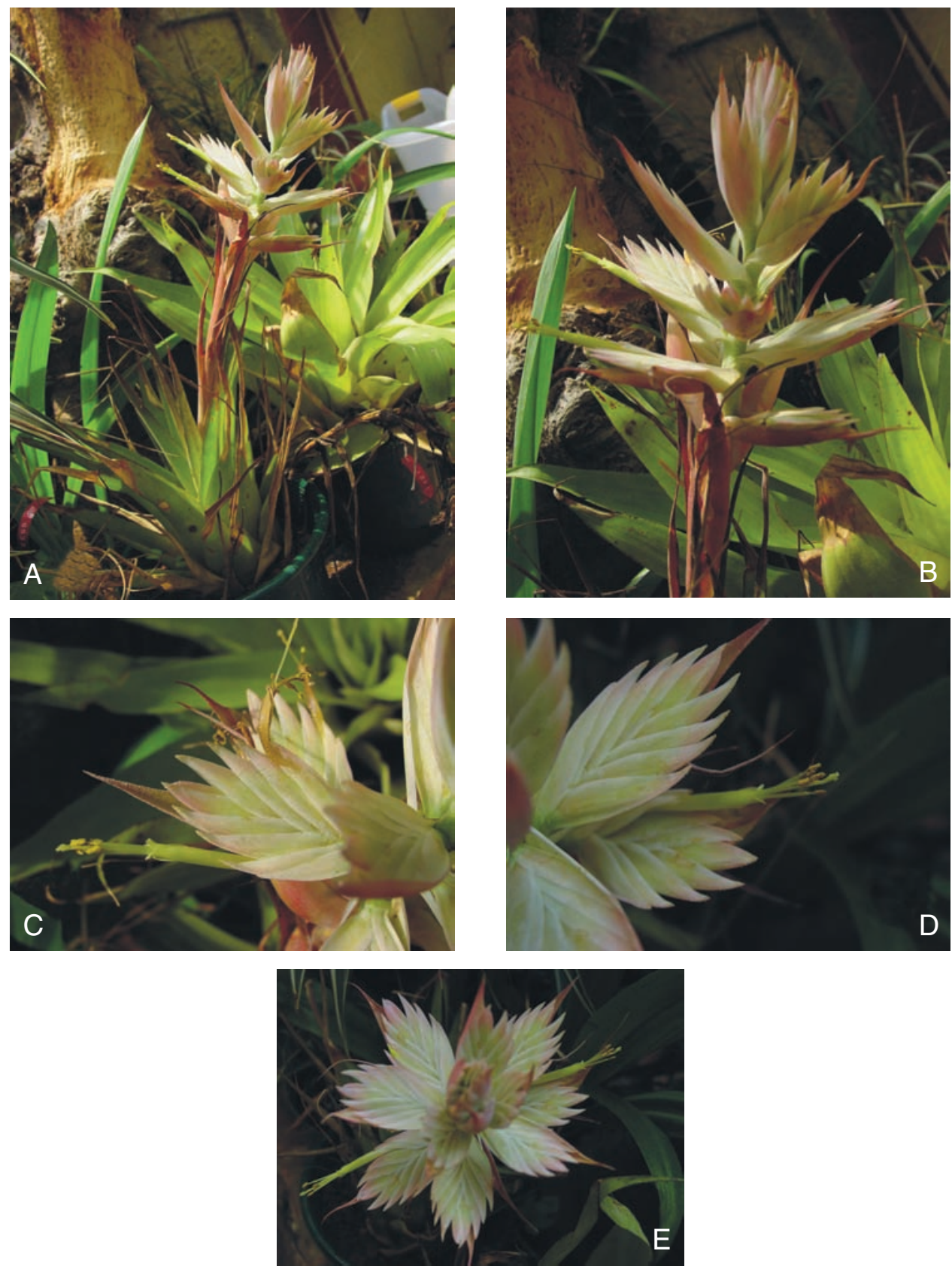

Fig. 2. Tillandsia sierrahalensis Espejo et López-Ferrari. A. hábito; B. inflorescencia, vista lateral; C. y D. detalle de las espigas; E. infloresencia, vista apical. 
scapus inflorescentiae erectus vel paulo curvatus, teres, 30-35 longus; inflorescentia bipinnata; spicae 9-11, ellipticae vel ovato-ellipticae, fortiter complanatae, $4-5.5 \mathrm{~cm}$ longae, 2.4-2.7 cm latae; bracteae florales distichae, imbricatae, ovato-triangulares, 3-4.7 cm longae, 1.3-1.5 cm latae, carinatae, glabrae, roseo-virides, nervatae; petala 5-5.2 cm longa, 6-7 mm lata, pallide viridia; stamina et stylus exserta.

Hierbas epífitas, acaules, de 50 a $60 \mathrm{~cm}$ de alto incluyendo la inflorescencia, arrosetadas, la roseta obcónica, de 25 a $35 \mathrm{~cm}$ de diámetro; hojas numerosas, vainas oblongas a oblongo-elípticas, de 9.5 a $11 \mathrm{~cm}$ de largo, de 5.5 a $6 \mathrm{~cm}$ de ancho, pardas claras, ligeramente discoloras con respecto a la lámina, particularmente en la superficie adaxial; láminas angostamente triangular-lanceoladas, erectas, de 18 a $25 \mathrm{~cm}$ de largo, de 2 a $2.5 \mathrm{~cm}$ de ancho, atenuadas, enteras, ligeramente coriáceas, densamente punctulado-lepidotas en ambas superficies; escapo rollizo, de 30 a $35 \mathrm{~cm}$ de largo, de 4.5 a $5 \mathrm{~mm}$ de diámetro, más largo que las hojas, erecto a algo arqueado; brácteas del escapo foliáceas, imbricadas y cubriéndolo totalmente, de 18 a $23 \mathrm{~cm}$ de largo; inflorescencia bipinnada, con 9 a 11 espigas ascendentes a divaricadas, el raquis conspicuamente acostillado cuando seco; brácteas primarias rosadas, ovadotriangulares, las inferiores foliáceas, de 4.5 a $11 \mathrm{~cm}$ de largo, de 2.5 a $3 \mathrm{~cm}$ de ancho, largamente atenuadas a acuminadas en el ápice; espigas elípticas a ovado-elípticas, de 4 a $5.5 \mathrm{~cm}$ de largo, de 2.4 a $2.7 \mathrm{~cm}$ de ancho, fuertemente comprimidas, con 4 a 7 flores, pedunculadas, los pedúnculos de $5 \mathrm{~mm}$ de largo, robustos; brácteas florales ovado-triangulares, de 3 a $4.7 \mathrm{~cm}$ de largo, de 1.3 a $1.5 \mathrm{~cm}$ de ancho, carinadas, glabras, conspicuamente nervadas cuando secas, acuminadas en el ápice, rosadas, verdosas hacia la base; sépalos angostamente elípticos a lanceolados, de 2.6 a $3 \mathrm{~cm}$ de largo, de 8 a 9 mm de ancho, agudos en el ápice, glabros, verdes, los dos adaxiales connados en la base por ca. $5 \mathrm{~mm}$ y fuertemente carinados, el anterior muy levemente carinado; pétalos oblongo-espatulados, de 5 a $5.2 \mathrm{~cm}$ de largo, de 6 a $7 \mathrm{~mm}$ de ancho, membranáceos, reflexos en el ápice, verdes pálidos con la base blanca; estambres exsertos, filamentos lineares, aplanados en la porción apical, de 6 a 6.3 cm de largo, blancos en la base, verdes pálidos en el ápice; anteras subbasifijas y versátiles, oblongas, de 3 a $3.2 \mathrm{~mm}$ de largo, negras; ovario ovoide, de ca. $8 \mathrm{~mm}$ de largo, de ca. $3.5 \mathrm{~mm}$ de diámetro, verde; estilo linear, de ca. $6 \mathrm{~cm}$ de largo, blanco a verde pálido; estigma conduplicado-espiral (tipo II sensu Brown \& Gilmartin, 1984), amarillo. Cápsula no vista.

Tipo: México, Jalisco, municipio de Tecalitlán, ca. 5-6 km después de Tecalitlán, rumbo a Jilotlán de los Dolores, 19²6'49" N, 103¹4'57" W, 1576 m s.n.m., 
bosque de pino-encino, colectado 8.IV.2003, prensado VIII.2003, A. Espejo, A. $R$. López-Ferrari, J. Ceja \& A. Mendoza R. 6533 (Holotipo: UAMIZ(x2)).

Tillandsia sierrahalensis se conoce hasta ahora sólo del estado de Jalisco (Fig. 3), en el municipio de Tecalitlán, en donde crece como epífita sobre Quercus spp. en bosques de pino-encino, en una altitud cercana a los $1500 \mathrm{~m}$. Florece en agosto.

La nueva especie presenta algunas similitudes con Tillandsia hintoniana L. B. Sm., conocida de los estados de México, Michoacán, Morelos y Guerrero (Espejo et al., 2004) (Fig. 3; Fig. 4), sin embargo es posible distinguirla claramente de la misma (Cuadro 1).

El nombre de la especie hace referencia a la Sierra del Halo, ubicada en el sureste de Jalisco, lugar en donde la especie fue colectada.

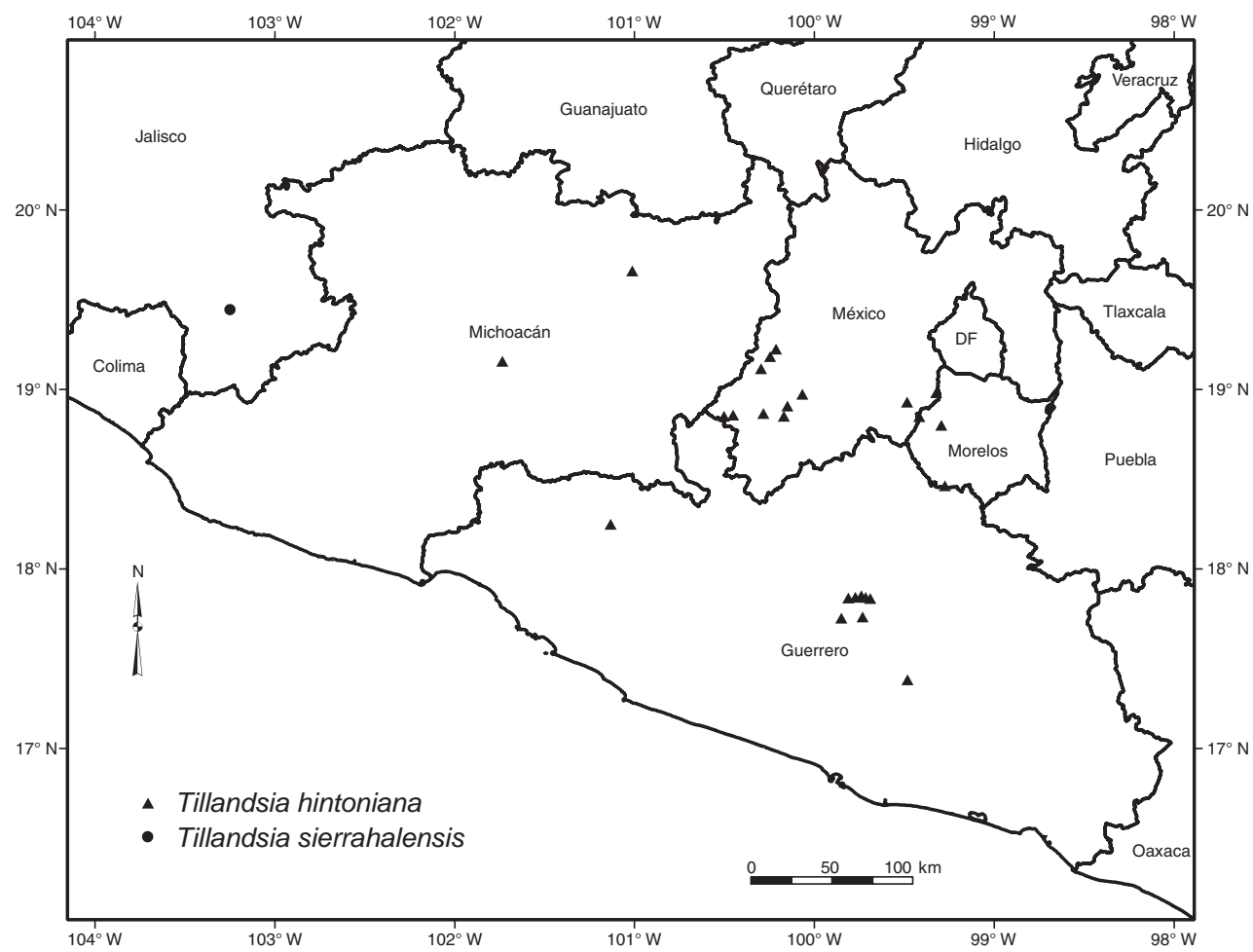

Fig. 3. Distribución de Tillandsia sierrahalensis Espejo et López Ferrari y de T. hintoniana L. B. Sm. 

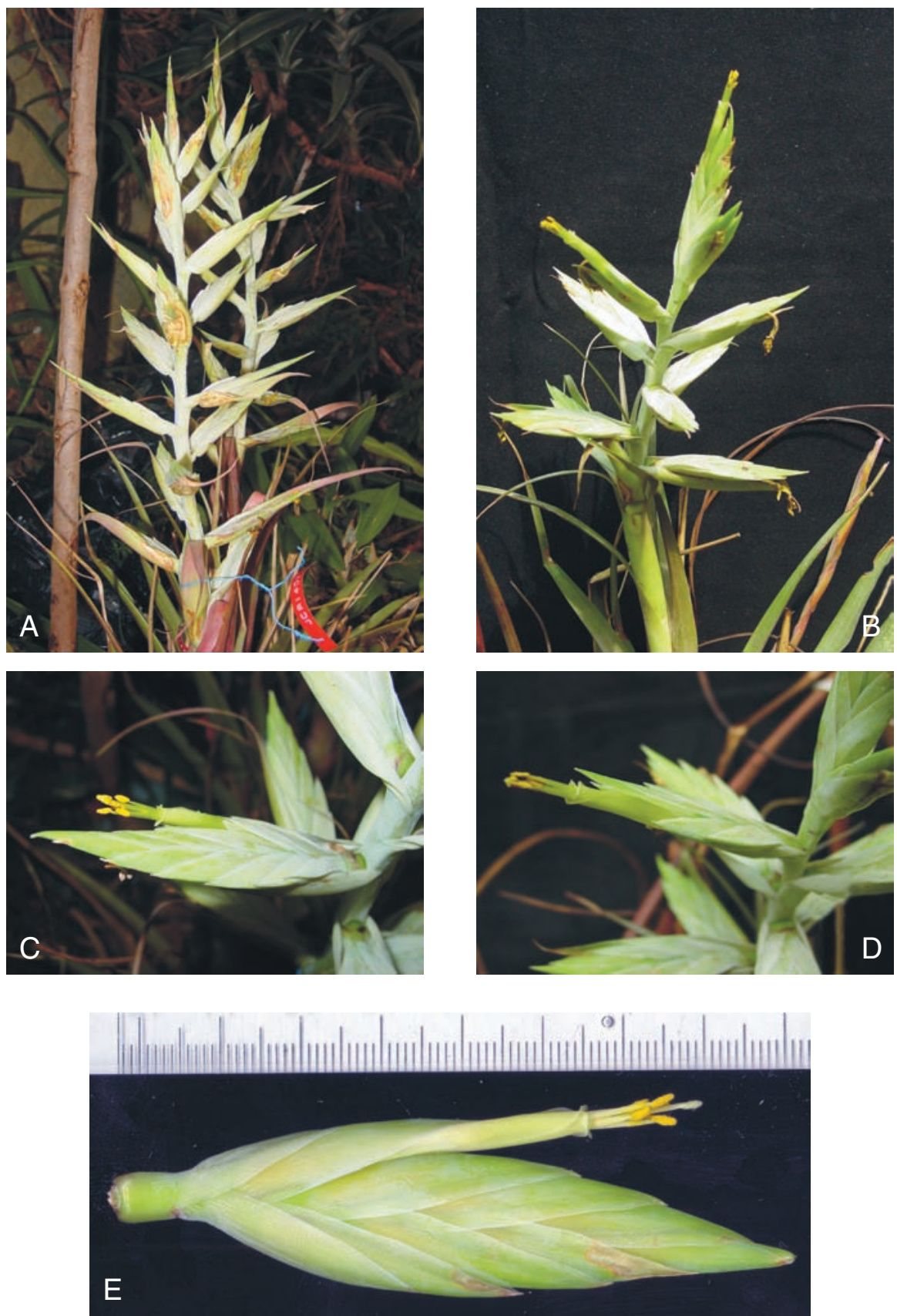

Fig. 3. Tillandsia hintoniana L. B. Sm. A. hábito; B. inflorescencia en vista lateral; C, D y E. detalle de las espigas. 
Cuadro 1. Caracteres comparativos de T. sierrahalensis Espejo et López Ferrari y T. hintoniana L. B. Sm.

\begin{tabular}{|l|l|l|}
\hline Caracteres & T. sierrahalensis & T. hintoniana \\
\hline Láminas foliares & $\begin{array}{l}\text { 18-25 cm de largo, erectas, más } \\
\text { cortas que el escapo } \\
\text { elípticas a ovado elípticas, de 4 a } \\
\begin{array}{l}\text { Espigas cm de largo, conspicuamente } \\
\text { pedunculadas, divaricadas a } \\
\text { ascendentes }\end{array}\end{array}$ & $\begin{array}{l}\text { 30-50 cm de largo, recurvadas en } \\
\text { el ápice, más largas que el escapo } \\
\text { lanceoladas, de 5 a } 8 \text { cm de largo, } \\
\text { divaricadas }\end{array}$ \\
\hline $\begin{array}{l}\text { Vainas de las } \\
\text { brácteas primarias }\end{array}$ & $\begin{array}{l}\text { tan largas o más largas que las } \\
\text { espigas, rosadas }\end{array}$ & más cortas que las espigas, verdes a \\
\hline Brácteas florales & $\begin{array}{l}\text { ovado-triangulares, de 3 a 4.7 cm } \\
\text { de largo }\end{array}$ & ovadas, de hasta 3 cm de largo \\
\hline Pétalos & de 5 a 5.2 cm de largo & de 4 cm de largo \\
\hline Distribución & Jalisco & Géxico, Michoacán, Morelos y \\
\hline
\end{tabular}

\section{AGRADECIMIENTOS}

Agradecemos a Javier García-Cruz la revisión crítica del manuscrito. Un especial reconocimiento a Jacqueline Ceja y Aniceto Mendoza por su incondicional e invaluable apoyo en el trabajo de campo y a Rolando Jiménez Machorro por la excelente ilustración de la nueva especie. Los curadores de los herbarios FCME, MEXU, SEL y UAMIZ nos dieron todas las facilidades para consultar sus colecciones, mil gracias. Parte de este trabajo pudo llevarse a cabo gracias al apoyo de la beca Elizabeth Bascom otorgada en el año 2004 por el Missouri Botanical Garden a A. R. López-Ferrari.

\section{LITERATURA CITADA}

Brown, G. K. \& A. J. Gilmartin. 1984. Stigma structure and variation in Bromeliaceae neglected taxonomic characters. Brittonia 36: 364-374.

Espejo, A., López-Ferrari, A. R., Ramírez-Morillo, I., Holst, B. K., Luther, H. E. \& W. Till. 2004. Checklist of Mexican Bromeliaceae with notes on species distribution and levels of endemism. Selbyana 25: 33-86. 


\section{ANEXO}

Exsiccata revisados

Tillandsia hintoniana L. B. Sm. Fig. 3

Guerrero: municipio de Chilpancingo de los Bravo, $20 \mathrm{~km}$ from Chilpancingo, ca. $4000 \mathrm{ft}$, P. Koide 2 (SEL); municipio de Eduardo Neri, $3.5 \mathrm{~km}$ al W de Carri-

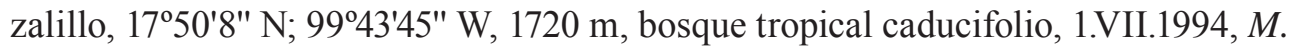
A. Monroy de la R. 257 (FCME), 10 km al W de Carrizalillo, 17²51'4" N; 9945'27" W, 1650 m, bosque de Quercus, 19.II.1995, M. Luna Flores 797 (FCME), al NE de Xochipala y El Mirabal, 1785 m, bosque de Quercus, 15.III.2002, O. A. López Sandoval 12 (FCME), $6 \mathrm{~km}$ al SE de Amatitlán, camino a Carrizalillo, 1750'8" N; 9944'43" W, 1705 m, bosque de Quercus, 9.IX.1993, R. Cruz Durán \& M. E. García G. 126 (FCME, MEXU), Amatitlán, $0.5 \mathrm{~km}$ al SE, camino a Carrizalillo, 17²51'40" N; 99045'19" W, 1570 m, bosque de Quercus, 1.VII.1994, R. Cruz Durán \& M. E. García G. 161 (FCME), cañada Carrizalillo, 2 km al ESE de Amatitlán, 1751'28" N; 9944'29" W, 1560 m, bosque de Quercus ecotonía con bosque tropical caducifolio, 11.III.1995, R. Cruz Durán \& M. E. García G. 767 (FCME); municipio de Leonardo Bravo, $0.5 \mathrm{~km}$ al oeste de La Escalera, 17²4'41" N; 9951'04" W, $1600 \mathrm{~m}$, bosque tropical caducifolio, 2.V.1998, J. Calónico S. 8703 (FCME); municipio de Zirándaro, $17 \mathrm{~km}$ al SE de Guayameo, 1140 m, encinar, 16.IV.1986, J. C. Soto N., S. Román G. \& F. Solórzano G. 12442 (MEXU). Estado de México: municipio de Ixtapan del Oro, $12 \mathrm{~km}$ sobre la desviación a Colorines, a partir de Valle de Bravo, 19¹3'30" N; $100^{\circ} 12^{\prime} 48^{\prime \prime} \mathrm{W}, 1775 \mathrm{~m}$, bosque de encino seco con elementos de selva baja, 6.III.1992, A. Espejo, A. R. López-Ferrari \& M. Flores C. 4693 (UAMIZ); municipio de Malinalco, entre Malinalco y Chalma, 1300 m, en barranca húmeda, 22.IV.1954, E. Matuda \& colaboradores 32175 (Paratipo: US); municipio de Otzoloapan, Otzoloapan, Dist. Valle de Bravo, 1300 m, en ladera húmeda matorral bajo, 5.IX.1954, E. Matuda \& colaboradores 31447 (US); municipio de San Simón de Guerrero, ca. 2 km después de Río Chilero rumbo a Tejupilco, 1858'29" N; 10003'57" W, 1730 m, bosque de pino-encino, 23.VIII.2003, J. Ceja \& A. Mendoza R. 1503 (UAMIZ(x2)); municipio de Santo Tomás, Ixtapantongo hasta La Junta, 600-800 m, en ladera seca, matorral alto, 24-25.IV.1954, E. Matuda \& colaboradores 30662 (Paratipos: MEXU, US); municipio de Tejupilco, Tejupilco, 18.IV.1935, G. B. Hinton et al. 7636 (Holotipo: US), 3 km adelante de la desviación a Palmar Chico, carretera El Estanco-Cañadas de Nanchititla, 1852'04" N; 100¹7'03" W, 625 m, selva baja, 11.III.1992, A. 
R. López-Ferrari \& A. Espejo 1629 (UAMIZ), Peñas Grandes (Largas), 10 km sobre el camino Cañadas Nanchititla-La Piaxtlera, $18^{\circ} 50^{\prime}$ N; $100^{\circ} 30^{\prime}$ W, ca. 2000 m, bosque de pino-encino, 11.III.1992, A. R. López-Ferrari \& A. Espejo 1646 (UAMIZ), en el Cerro de la Muñeca, 1500 m, ladera seca, rocosa, 27-28.II.1954, E. Matuda \& colaboradores 30516 (Paratipo: US(x2)); Cañada de Nanchititla, 1600 m, ladera húmeda, bosque mixto de encinos y pinos, 25-26.V.1954, E. Matuda \& colaboradores 30791 (Paratipos: MEXU, US). Michoacán: municipio de Charo, parque nacional José María Morelos y Pavón, aproximadamente $1 \mathrm{~km}$ antes de Pontezuelos, carretera Morelia-Ciudad Hidalgo, 19³9'39" N; 10100'44" W, 2065 m, bosque de encinopino, 10.IV.1992, A. Espejo, A. R. López-Ferrari \& M. Flores C. 4752 (UAMIZ(x2)); municipio de Nuevo Urecho, 1 km sobre la desviación a Nuevo Urecho, a partir del camino Ario de Rosales-La Huacana, 1909'30" N; 10144'06" W, 1600 m, matorral espinoso alto, con leguminosas y burseras, 20.VI.1991, A. Espejo, A. R. López-Ferrari \& M. Flores C. 4460 (UAMIZ). Morelos: municipio de Miacatlán, 3.8 km después de Palpan rumbo a Palo Grande, 1850'58" N; 99 $25^{\circ} 03^{\prime \prime} \mathrm{W}, 1561 \mathrm{~m}$, selva baja caducifolia, 15.II.2003, J. Ceja \& A. Mendoza R. 1401 (MEXU(x2), UAMIZ(x2)); municipio de Puente de Ixtla, $5 \mathrm{~km}$ sobre la brecha a El Salto, a partir de Tilzapotla, 18 $27^{\prime} 53^{\prime \prime} \mathrm{N} ; 9^{\circ} 16^{\prime} 32^{\prime \prime} \mathrm{W}, 1530 \mathrm{~m}$, bosque de encino con elementos de bosque tropical caducifolio, 9.XII.1999, A. Espejo, A. R. López-Ferrari, J. Ceja, A. Mendoza R. \& I. Ramírez M. 1050 (UAMIZ(x2)); municipio de Xochitepec, Xochicalco, 15.II.1866, L. Hahn s. n. (P); sin municipio indicado, arriba de Cuernavaca, 1900 m, bosque de encino, 17.V.1968, E. Matuda 38644 (MEXU).

Recibido en agosto de 2006. Aceptado en abril de 2007. 